\title{
Cell receptors and cell signalling
}

\author{
I J Uings, S N Farrow
}

\begin{abstract}
All cells in a multicellular organism are constantly exposed to a variety of extracellular signals that they need to interpret and translate into an appropriate response to their environment. These signals can be soluble factors generated locally (for example, synaptic transmission) or distantly (for example, hormones and growth factors), ligands on the surface of other cells, or the extracellular matrix itself. To achieve this, cells maintain a diversity of receptors on their surface that respond specifically to individual stimuli. These receptors fall into families, based primarily on the way in which they generate the intracellular signals that give rise to the particular functional responses. Moreover, the activity of a given receptor can be modulated by other signalling pathways in a variety of ways, generating the flexibility required of such a complex system. This review aims to describe the function of the major classes of receptor, including $G$ protein coupled receptors, receptor tyrosine kinases, ligand gated ion channels, integrins, and cytokine receptors, and to demonstrate the "crosstalk" that exists between these systems.

(F Clin Pathol: Mol Pathol 2000;53:295-299)
\end{abstract}

Keywords: cell signalling; cell receptors; cytokine receptors; receptor tyrosine kinases; ligand gated ion channels; G protein coupled receptors

The ability of cells to interact with and adapt to their environment is one of the fundamental processes of cell biology. This responsiveness is achieved predominantly through the expression at the cell surface of a repertoire of specific receptors that are sensitive to the composition of the surrounding environment. The signals that trigger these receptors can be presented in a variety of contexts-for example, soluble factors (chemicals, polypeptides, proteins, sugars, etc), a ligand bound to another cell, or the extracellular matrix itself. The receptors then transduce these extracellular signals across the plasma membrane and, through the activation of intracellular signalling pathways, bring about the appropriate functional response. Moreover, cells have developed sophisticated systems to integrate inputs from multiple signals.

In this review, we will discuss the various means by which different classes of cell surface receptors are triggered, the mechanisms by Dr Farrow snf8409@GlaxoWellcome.co.uk

Accepted for publication 22 June 2000 in which the activities of the receptors can be modulated. We begin by looking at the diverse family of cytokine receptors that play a key role in regulating the function of the haematopoietic system and in coordinating immune responses.

\section{Cytokine receptors}

PLEIOTROPY AND REDUNDANCY

Cytokines can exert effects on numerous cell types and can interact with, induce, or inhibit other cytokines, leading to the development of the concept of a cytokine network controlling those biological processes that involve cellular interactions. ${ }^{1}$ Cytokines can regulate diverse biological processes such as cell death and survival, cell proliferation, and cellular differentiation $^{2}$; moreover, different cytokines can act on the same cell type to induce a similar response. These abilities are dependent in part upon the multimeric nature of the cell receptors for cytokines, ${ }^{3}$ but also illustrate a fundamental issue in receptor mediated signalling, the means by which diverse extracellular stimuli are integrated into a coherent response.

\section{RECEPTOR CLASSIFICATION}

The molecular revolution in biology has allowed the cloning of numerous cytokines and their cognate receptors, and it has become clear that the receptors can be classified into families according to protein sequence homologies in their extracellular domains. ${ }^{4}$

One of the largest families comprises the type I or haematopoietin receptors, which respond to cytokines such as interleukin 2 (IL2), IL-4, IL-5, and IL-6, prolactin growth hormone, and granulocyte macrophage colony stimulating factor (GM-CSF). These receptors share features in two domains located in the extracellular portion. This homologous region consists of two globular structures that fold into a barrel-like structure, with a hinge region between them, which functions as a ligand binding pocket. ${ }^{5}$ All type 1 receptors contain a single membrane spanning domain and a cytoplasmic domain. The characterisation of the composition of type I receptors has led to a better understanding of the functional redundancy attributed to their cytokine ligands. High affinity receptors are made up of two chains, namely $\alpha$ and $\beta$. The $\alpha$ chain is the cytokine specific subunit, the $\beta$ chain alone cannot recognise a particular cytokine. However, it forms the high affinity receptor upon binding to the $\alpha$ chain, and is then responsible for transducing the signal. Thus, the fact that some receptors share the same $\beta$ chain can explain some of the coexistent biological activities of individual cytokines. The multimeric composition of this family of receptors can therefore allow the classification of cytokines based on the presence of a common signal transducing element-for example, the gp140 subgroup 
which comprises the receptors for IL-3, GM-CSF, and IL-5. However, it should be noted that functional redundancy is also achieved by the use of common downstream signalling molecules, as will be described for the receptors for tumour necrosis factor (TNF) and IL-1.

Class II cytokine receptors show some structural similarities to type I, but differ in the presence of characteristic cysteine pairs located in the extracellular domain. A key member of this family is the receptor for interferon. ${ }^{6}$

Class III cytokine receptors comprise the proteins related to the TNF receptor. ${ }^{78}$ This large and important family is characterised primarily by the presence of multiple cysteine rich extracellular domains, varying from two to six repeats. However, the family can be subdivided into two groups: those without appreciable homology in the cytoplasmic domains (such as the receptors for nerve growth factor (NGF) and CD40) and those that contain an 80 amino acid cytoplasmic "death domain". So far, only one member of the family, namely CD120a, has been subjected to crystallographic analysis, the results of which support the hypothesis that a trimeric ligand complex binds to three receptor molecules.

Many of the members of this family exist in soluble forms; indeed osteoprotegrin, the focus of much recent interest (as a result of its role in bone formation), exists only as a soluble molecule. ${ }^{9}$ These soluble receptors consist of the extracellular portion of the corresponding full length receptor, and seem to function by competing with the membrane bound receptor for soluble ligand. Interestingly, some viruses have exploited this inhibitory potential as a means of defence against the antiviral activities of TNF ligands. For example, poxviruses produce soluble forms of CD120b and CD $30 .{ }^{10}$

\section{SIGNALLING MOTIFS}

The subfamily of death domain containing receptors has been the focus of much recent research, stimulated by the biological importance of cytokines such as TNF in the regulation of inflammatory processes. Production of and signalling by TNF is believed to play a key role in diseases such as rheumatoid arthritis, and a very recent clinical breakthrough has been made through the use of a soluble TNF receptor molecule to block the normal signalling induced by TNF itself. A related ligand, TRAIL, is also in clinical trials as an anticancer agent, based on the observation that its receptor appears to be expressed selectively on tumour cells. ${ }^{11}$

The mechanism by which this family activates downstream signal transduction has been studied extensively. The unactivated receptor is induced to trimerise by the binding of ligand, also acting as a trimer. This trimeric receptor then recruits several signalling and adaptor molecules, collectively called the DISC complex, originally characterised for the FAS death receptor. ${ }^{12}$ The DISC complex is then able to activate signal transduction cascades leading typically to activation of the transcription factors $\mathrm{NF}-\kappa \mathrm{B}$ and $\mathrm{AP}-1$, and also in many cases to the induction of apoptosis. Serine/ threonine protein kinases are key components of these intracellular pathways, and the induction of apoptosis results from the activation of a cascade of a newly characterised family of cysteine proteases, the caspases. ${ }^{13}$ Feedback mechanisms also operate to induce downregulation of the receptor at the cell surface, although this process is not so well characterised.

A fourth class of cytokine receptors is exemplified by the IL-1 receptor, ${ }^{14}{ }^{15}$ the extracellular domain of which contains three immunoglobulin-like regions. The intracellular portions of these type IV receptors show a degree of similarity, and recent work has begun to characterise the signalling complex formed by the activated IL-1 receptor. As noted above, although the TNF and IL-1 receptors are members of different families, the signal transduction pathways they initiate converge such that several downstream signalling molecules are shared, notably a newly characterised complex of kinases, the IKKs. ${ }^{16}$

\section{Cell receptors and the extracellular matrix}

Thus far, we have focused on the regulation of crosstalk between cells and the key role played by cytokine receptors; however, cell survival and proliferation require contact with the extracellular matrix, an interaction that is regulated primarily by another family of cell surface receptors, the integrins. ${ }^{17}{ }^{18}$ The extracellular matrix exerts profound control over cells; integrins function to attach cells to the matrix and mediate mechanical and chemical signals. ${ }^{19}$ Growth factor receptors and ion channels are regulated by these signals, which converge on diverse functions such as apoptosis, proliferation, and differentiation.

Integrin receptors are composed of two subunits, namely $\alpha$ and $\beta$; each $\alpha \beta$ combination has its own binding specificity and signalling properties. Most recognise several extracellular matrix proteins, and conversely several matrix proteins such as fibronectin and collagens bind to several integrins. Integrin extracellular binding activity is regulated from the inside of the cell, whereas binding of the extracellular matrix induces signals that are transmitted into the cell.

The cytoplasmic portions of integrins are generally short and are without intrinsic enzymatic activity. Consequently, the signals are transduced by associating with adaptor proteins such as cytoplasmic kinases and transmembrane growth factor receptors. Various kinase families can be activated, including tyrosine kinases, such as FAK and Fyn, and src family kinases. ${ }^{19}$ As the integrins bind to the extracellular matrix they cluster in the plane of the cell membrane, an event that results in the assembly of actin filaments. This reorganisation of actin filaments into larger stress fibres in turn causes more integrin clustering, creating a positive feedback system. Consequently, extracellular matrix proteins, integrins, and cytoskeletal proteins assemble into aggregates on 
either side of the membrane, which can be visualised by confocal microscopy.

The cooperation between integrins and growth factor receptors is necessary for optimal activation. For example, the receptor for insulin undergoes maximal activation only under appropriate cell attachment conditions. It also appears that certain integrins can preferentially associate with particular growth factor receptors. Growth factor receptors themselves constitute a large and important family and illustrate several interesting additional principles.

\section{Receptor tyrosine kinases}

Polypeptide growth factors such as epidermal growth factor (EGF), ${ }^{20}$ and platelet derived growth factor (PDGF) ${ }^{21}$ exert profound effects on cellular proliferation, maturation, and function, particularly during development and in the response to injury in the adult. Malfunction or dysregulation of these powerful systems contributes to the development of many neoplastic growths, where they provide a persistent mitogenic signal for the transformed cells. ${ }^{21-23}$ The growth factor receptors form a family that has intrinsic protein tyrosine kinase activity in the cytoplasmic domains. ${ }^{24}$ The receptors all have a single membrane spanning helix and an extracellular ligand binding domain of diverse structure. ${ }^{25}$ When a growth factor binds to its receptor, it induces both receptor dimerisation and an increase in the activity of the kinase. The intracellular region of the activated receptor then becomes extensively autophosphorylated, and these phosphorylated tyrosines form specific docking sites for intracellular molecules that contain $\mathrm{SH} 2$ domains. In this way, a large signalling complex is assembled rapidly around activated receptors, from which a variety of signal transduction cascades emanate. For instance, in the activated PDGF $\beta$ receptor, two phosphotyrosine residues close to the membrane mediate binding of Src family kinases and STAT (signal transducer and activator of transcription) molecules, two sites at the C-terminus recognise phospholipase $\mathrm{C} \gamma$ and the tyrosine phosphatase SHP-2, and sites in the middle of the cytoplasmic domain recruit phosphatidyl inositol 3-kinase, the GTPase activating protein of ras, and adaptors such as Grb2, Nck, and Shc. ${ }^{26}$ Many of these proteins are substrates for the receptor kinase and become phosphorylated. The multiplicity of components rapidly activated in this way underpins the profound and diverse effects growth factors such as PDGF have on their target cells because each signalling pathway that is activated can initiate or amplify one or more functional responses. ${ }^{26}$ Moreover, many growth factor ligands induce heterodimerisation of multiple receptors, and the signalling pathways and functional responses subsequently activated change with receptor composition, ${ }^{27}$ providing a mechanism to generate a large number of different cellular responses from a limited number of receptors.

Concomitant with the initiation of signal transduction, activated receptors are internal- ised rapidly by the endocytic pathway and subsequently degraded in the lysosomes. ${ }^{26}{ }^{28} \mathrm{In}$ addition, receptor phosphorylation appears to acts as a signal for ubiquitinylation, thus promoting degradation of receptors by a proteasome dependent pathway. ${ }^{26}$ Consequently, ligand binding causes a downregulation of surface receptors and therefore an attenuation of the signal transduction cascades, thus limiting the degree of cellular activation.

\section{Ligand gated ion channels}

The maintenance of the cell membrane as a barrier to the flux of ions plays a central role in cellular physiology, particularly in the nervous system. Therefore, it is not surprising that many receptors function by altering the permeability of membranes to specific ions. Certain receptors in the central nervous system, such as the $\mathrm{GABA}_{\mathrm{A}}, 5 \mathrm{HT}_{3}$, glycine, and nicotinic acetylcholine (nACh) receptors all function in a similar fashion to ligand gated ion channels. $^{30}$ These receptors are pentameric complexes, assembled from several similar (in some cases identical) subunits, each of which has a large extracellular (synaptic) region, several membrane spanning regions, and small intracellular domains. A single transmembrane $\alpha$-helix from each subunit is used to form a central pore through which the ions pass. ${ }^{31}$ The glycine receptor has an additional cytoplasmic component, gephyrin, which links the receptor to the cytoskeleton and other signalling components. $^{32}$ Ligand binding occurs on the synaptic domain of one or more of the subunits, and induces a conformational change in the receptor from a "closed" state, which is impermeable to ions, into an "open" state, allowing ions to flow through the central pore. This is followed by a rapid transition to a third state, referred to as "desensitised", where the channel is closed and the receptor unresponsive to further stimulation. This desensitised channel then slowly resets into the closed state ready for reactivation.

By virtue of the size and charge of residues lining the pore, different receptors show selective permeability for different ions. The glycine and $\mathrm{GABA}_{\mathrm{A}}$ receptors are anion channels that, when open, allow the movement of chloride ions. In contrast, the nACh receptor is selectively permeable to monovalent cations such as sodium. The flow of ions can initiate neuron depolarisation and hence activation (for example, the $\mathrm{nACh}$ receptor), or can decrease the responsiveness of the synapse (for example, the glycine receptor)..$^{33}$

The activity of ligand gated ion channels can be altered in several ways. Allosteric modulators (such as zinc ions for the glycine receptor) can increase or decrease the apparent affinity of the receptors for their cognate ligands. ${ }^{33}$ More interestingly, the cytoplasmic portion of the receptors can be phosphorylated by a variety of kinases including protein kinase A (PKA), PKC, calmodulin dependent kinase II (CAMKII) and Src. The functional consequences of this phosphorylation are diverse. Serine phosphorylation of the nACh receptor by PKA appears to increase the rate of receptor desen- 
sitisation. In contrast, tyrosine phosphorylation of the glutamate receptor enhances the functioning of the channel. ${ }^{34}{ }^{35}$ Thus, prior activation of intracellular signalling pathways in response to other stimuli can modulate the sensitivity of neurons to ligands such as glycine or ACh. This complexity might be the key to synaptic plasticity, and serves to illustrate the way in which cells use signal transduction pathways to integrate the set of signals that they receive from their environment into an appropriate coordinated cellular response.

\section{G protein coupled receptors}

The $G$ protein coupled seven transmembrane proteins (GPCs) form one of the largest families of cell surface receptors. They share a consistent general topology, with an N-terminal extracellular domain, seven membrane spanning helices separated by loop regions of varying sizes, and an intracellular C-terminal domain. GPC receptors activate signal transduction through the coupling of their cytoplasmic domains to a family of heterotrimeric GTP binding proteins ( $\mathrm{G}$ proteins). Ligand binding promotes the exchange of $G$ protein bound GDP for GTP, and this activated G protein leaves the receptor complex and initiates signal transduction. The GTP is hydrolysed to GDP by the intrinsic GTPase activity of the G protein itself, providing a convenient self limiting mechanism. However, each receptor can activate a large number of $\mathrm{G}$ proteins before the signal is terminated by receptor internalisation, ensuring substantial amplification of the signal before termination. Different GPC receptors use the same basic mechanism to act on a wide variety of signal transduction pathways, including adenyl cyclase, tyrosine kinase cascades, and phospholipases.

The ligands for these receptors are diverse and they mediate a large number of cell responses as varied as retinal stimulation by light, the control of heart rate, and cellular migration. ${ }^{36}$ To illustrate this diversity we will briefly examine two emerging areas of general interest; namely, mechanisms of odorant and chemosensory receptors and chemokine receptors.

Chemosensation in higher organisms consists of taste and smell. In mammals, olfactory sensory neurons are located in the nasal cavity. Odorants activate specific receptors on the cilia of these neurons. These molecular receptors are invariably $G$ protein coupled seven transmembrane proteins.$^{37}$ In the mouse and rat, the repertoire of odorant receptors is estimated to be about $1 \%$ of the genome, more than the $\mathrm{T}$ cell receptor and immunoglobulin families combined. These genes were first identified by exploiting several features and assumptions about their likely structure, in particular the conservation of certain amino acid motifs within the seven transmembrane region. However, compared with the other gene families already discussed, information about these genes is still fragmentary. Recent genetic manipulation experiments have suggested that, in vertebrates, odorant receptors might play a dual role as both chemosensors and as molecu- lar addressers that guide axons of olfactory sensory neurons to their precise target in the brain.

Chemokines are small proteins that function as chemoattractants for haematopoietic cells and are particularly involved in the coordination of immune and inflammatory responses. $^{38} 39$ There are two principal classes of chemokines, defined by the spacing of cysteine residues as CC and CXC chemokines. The CC chemokines include RANTES, eotaxin, monocyte chemoattractant protein 1 (MCP-1), and macrophage inflammatory protein $1 \alpha$ (MIP$1 \alpha$ ), whereas the CXC chemokines are exemplified by IL- 8 and Gro- $\alpha$. There is an increasing number of chemokine receptors, all of which appear to be particularly promiscuous, with each receptor binding to multiple ligands and most of the chemokines using a variety of receptors. Thus, RANTES will activate CCR1, CCR3, and CCR5, whereas CCR1 will bind RANTES, MCP-1 and MIP-1 $\alpha$. However, there is no crossreactivity between the CC and CXC families. ${ }^{40}$

The chemokine receptors are classic GPC receptors, functioning through heterotrimeric $\mathrm{G}$ proteins. Activated chemokine receptors are internalised rapidly by an endosomal pathway, leading to desensitisation. Moreover, unlike many cytokine receptors, the repertoire of chemokine receptor expression at the surface of any one cell type is particularly variable, being sensitive to many kinds of cellular activation. Thus, the responsiveness of cells to chemokines is very dependent on the environment to which they have been exposed. This is exactly what one would expect, given their role as part of the complex coordination of immune responses, and illustrates another mechanism that cells use to adapt their behaviour according to multiple inputs.

\section{Summary and perspectives}

In this review we have described how the major classes of cell surface receptor mediate the transduction of an extracellular stimulus into intracellular events that change the behaviour of the cell. We have not discussed every receptor or mechanism, but have tried to illustrate how different signals become integrated into a coordinated response by the complex interplay of signal transduction pathways and the receptors that initiate them.

The availability of gene sequences generated by the human genome project has had a massive impact on the study of cell receptors and cell signalling, as seen with the classification of many of the receptor families discussed in this review. However, as completion of the project approaches, the focus will inevitably shift towards understanding the fundamental biology and biochemistry of the thousands of recently discovered proteins. A key incentive for this is illustrated by the fact that many of the cell receptors and signalling pathways discussed are the target of intensive medical and pharmaceutical research, and there can be little doubt that the first decade of the new century will see unprecedented advances in these fields. 
1 Fernandez-Botran R, Chilton PM, Ma Y. Soluble cytokine Fernandez-Botran R, Chilton PM, Ma Y. Soluble cytokine receptors: their roles in immunoregulation

2 Orkin SH. Development of the hematopoietic system. Curr Opin Genet Dev 1996;6:597-602.

3 Bazan JF. Structural design and molecular evolution of a cytokine receptor superfamily. Proc Natl Acad Sci US A 1990;87:6934-8.

4 Campbell ID. The modular architecture of leukocyte cell-surface receptors. Immunol Rev 1998;163:11-80.

5 Theze J, Alzari PM, Bertoglio J. Interleukin 2 and its receptors: recent advances and new immunological functions. Immunol Today 1996;17:481-6.

6 De Maeyer E, Maeyer-Guignard J. Type I interferons. Int Rev Immunol 1998;17:53-73.

7 Wallach D, Varfolomeev EE, Malinin NL, et al. Tumor necrosis factor receptor and Fas signalling mechanisms. Annu Rev Immunol 1999;17:331-67.

8 Smith CA, Farrah T, Goodwin RG. The TNF receptor superfamily of cellular and viral proteins: activation, superfamily of cellular and viral proteins:
costimulation, and death. Cell 1994;76:959-62.

9 Emery JG, McDonnell P, Burke MB, et al. Osteoprotegerin is a receptor for the cytotoxic ligand TRAIL. F Biol Chem 1998:273:14363-7.

10 McFadden G, Graham K, Ellison K, et al. Interruption of cytokine networks by poxviruses: lessons from myxoma virus. F Leukoc Biol 1995;57:731-8

11 Golstein P. Cell death: TRAIL and its receptors. Curr Biol 1997;7:R750-3

12 Nagata S. Fas ligand-induced apoptosis. Annu Rev Genet 1999;33:29-55.

13 Cohen GM. Caspases: the executioners of apoptosis. Biochem f 1997;326:1-16.

14 Dinarello CA. Interleukin-1. Adv Pharmacol 1994;25:2151.

15 Colotta F, Dower SK, Sims JE, et al. The type II "decoy" receptor: a novel regulatory pathway for interleukin 1 . Immunol Today 1994;15:562-6.

16 Zandi E, Karin M. Bridging the gap: composition, regulation, and physiological function of the IkappaB regulation, and physiological function of the

17 Aota S, Yamada KM. Integrin functions and signal transduction. Adv Exp Med Biol 1997;400B:669-82.

18 Yamada KM, Geiger B. Molecular interactions in cell adhesion complexes. Curr Opin Cell Biol 1997;9:76-85.

19 Schlaepfer DD, Hunter T. Integrin signalling and tyrosine phosphorylation: just the FAKs? Trends Cell Biol 1998;8: 151-7.

20 Wells A. EGF receptor. Int F Biochem Cell Biol 1999;31:637-

21 Heldin $\mathrm{CH}$, Westermark B. Mechanism of action and in vivo role of platelet-derived growth factor. Physiol Rev 1999;79: $1283-316$
22 Ebert AD, Wechselberger C, Martinez-Lacaci I, et al. Expression and function of EGF-related peptides and their receptors in gynecological cancer-from basic science to therapy. F Recept Signal Transduct Res 2000;20:1-46.

23 Khazaie K, Schirrmacher V, Lichtner RB. EGF receptor in neoplasia and metastasis. Cancer Metastasis Rev 1993;12: 255-74.

24 Ullrich A, Schlessinger J. Signal transduction by receptors with tyrosine kinase activity. Cell 1990;61:203-12.

25 Hubbard SR. Structural analysis of receptor tyrosine kinases. Prog Biophys Mol Biol 1999;71:343-58.

26 Heldin $\mathrm{CH}$, Ostman A, Ronnstrand L. Signal transduction via platelet-derived growth factor receptors. Biochim Biophys Acta 1998;1378:F79-113.

27 Riese DJ, Stern DF. Specificity within the EGF family/ErbB receptor family signalling network. Bioessays 1998;20:41-8.

28 Lamaze C, Schmid SL. Recruitment of epidermal growth factor receptors into coated pits requires their activated tyrosine kinase. f Cell Biol 1995;129:47-54.

29 Galcheva-Gargova Z, Theroux SJ, Davis RJ. The epidermal growth factor receptor is covalently linked to ubiquitin. Oncogene 1995;11:2649-55.

30 Unwin N. Neurotransmitter action: opening of ligand-gated ion channels. Cell 1993;72:31-4.

31 Sansom MS, Adcock C, Smith GR. Modelling and simulation of ion channels: applications to the nicotinic acetylcholine receptor. F Struct Biol 1998;121:246-62.

32 Vannier C, Triller A. Biology of the postsynaptic glycine receptor. Int Rev Cytol 1997;176:201-44.

33 Rajendra S, Lynch JW, Schofield PR. The glycine receptor. Pharmacol Ther 1997;73:121-46.

34 Swope SL, Moss SI, Raymond LA, et al. Regulation of ligand-gated ion channels by protein phosphorylation. $A d v$ Second Messenger Phosphoprotein Res 1999;33:49-78.

35 Swope SL, Moss SJ, Blackstone CD, et al. Phosphorylation of ligand-gated ion channels: a possible mode of synaptic plasticity. FASEB F 1992;6:2514-23.

36 Kobilka B. Adrenergic receptors as models for $\mathrm{G}$ proteincoupled receptors. Annu Rev Neurosci 1992;15:87-112.

37 Buck L, Axel R. A novel multigene family may encode odorant receptors: a molecular basis for odor recognition. Cell 1991;65:175-87.

38 Homey B, Zlotnik A. Chemokines in allergy. Curr Opin Immunol 1999;11:626-34.

39 Wells TN, Proudfoot AE, Power CA. Chemokine receptors and their role in leukocyte activation. Immunol Lett 1999;65:35-40.

40 Murphy PM, Baggiolini M, Charo IF, et al. International union of pharmacology. XXII. Nomenclature for chemokine receptors. Pharmacol Rev 2000;52:145-76. 